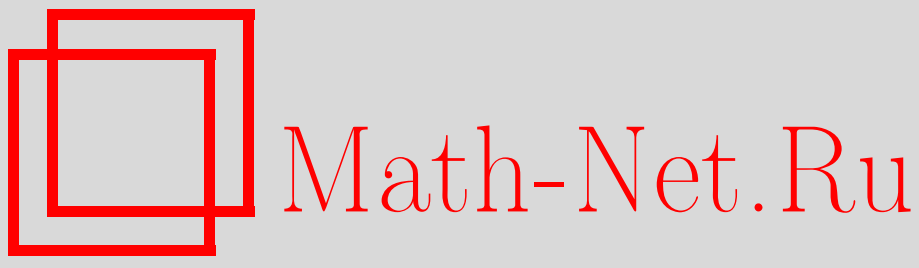

Е. И. Бережной, В. И. Буренков, Улучшенные интерполяционные теоремы для одного класса операторов, Изв. РАН. Сер. матем., 1998, том 62, выпуск 4, 3-24

DOI: https://doi.org/10.4213/im195

Использование Общероссийского математического портала Math-Net.Ru подразумевает, что вы прочитали и согласны с пользовательским соглашением http://www. mathnet.ru/rus/agreement

Параметры загрузки:

IP : 54.162 .27 .143

26 апреля 2023 г., $14: 16: 55$ 
УДК $513.88+517.5$

\author{
Е.И. Бережной, В. И. Буренков
}

\title{
Улучшенные интерполяционные теоремы для одного класса операторов
}

\begin{abstract}
Для класса операторов, переводящих конус положительных функций в конус монотонно убывающих функций, приведены новые теоремы интерполяции, основанные на понятии $K^{c}$-фукционала. Предложена формула для вычисления $K^{c}$-функционала для некоторых пар пространств. Приведен пример интерполяционной пары пространств, для которой конусы, получаемые с помощью $K$-функционала Питре и $K^{c}$-функционала, различны.

Библиография: 16 наименований.
\end{abstract}

\section{$\S 1$. Введение}

Теория интерполяции линейных операторов, начиная от пионерских работ Рисса, Торина и Марцинкевича [1]-[5], интерполирует основное свойство линейного оператора - ограниченность нормы.

В дальнейшем неоднократно предпринимались попытки проинтерполировать более тонкие свойства операторов. Например, успешно удалось проинтерполировать различные количественные характеристики свойства компактности [5, с. 122]. С другой стороны, представляет интерес при рассмотрении некоторых специальных классов операторов с дополнительными свойствами попытка улучшить общие интерполяционные теоремы для этих специальных классов. К этому вопросу и относится настоящая работа, которая посвящена теоремам интерполяции для специального класса линейных операторов в идеальных пространствах, названного в работе классом $B$ и содержашего, например, операторы интегрирования, вольтерровские с положительньми и монотонными ядрами и близкие к ним.

Операторы этого класса нашли различное применение в гармоническом анализе (см., например, [6]-[9]). Они обладают замечательным свойством: переводят положительные функции в монотонные. Поэтому, с одной стороны, их ограниченность можно проверять лишь на положительных функциях, а с другой стороны, образ конуса положительных функций идеального пространства будет содержаться в конусе монотонных функций. Используя это наблюдение, в работе вводится понятие $K^{c}$-функционала, определенного для монотонных функций и построенного по разложению их по монотонным же функциям, и приводится формула для его вычисления в некоторых идеальных пространствах. На основе этого вычисления приведен пример интерполяционной пары идеальных пространств, когда классический $K$-функционал Питре и $K^{c}$-функционал, введенный в работе, не эквивалентны. Исходя из этого приведен пример интерполяционной пары идеальных пространств, для которой интерполящионные пространства, получаемые с помощью

(С) Е.И. БЕРЕЖной, В.И. БУРЕнКОв 1998 
$K$-функционала Питре и введенного $K^{c}$-функционала, дают различные пространства, т.е. нормы на этих пространствах не эквивалентны. Для некоторых же классических пар идеальных пространств интерполяционные пространства, полученные с помошью $K$-функционала и $K^{c}$-функционала, совпадают.

\section{§ 2. Основные теоремы}

Прежде всего отметим, что термины "положительная функция $f$ " и "отрицательная функция $g$ " в настояшей работе означают, что $f(t) \geqslant 0$ и $g(t) \leqslant 0$, а термины "убываюшая функция $f$ " и "возрастаюшая функция $g$ " означают, что если $t \geqslant s$, то $f(t) \leqslant f(s)$ и $g(t) \geqslant g(s)$ соответственно. Аналогичные соглашения действуют и для других высказываний, содержащих термины "положительный", "отрицательный” и "убывающий", "возрастаюший”.

Приведем некоторые сведения из теории интерполяции линейных операторов и идеальных пространств.

Пусть $S\left(\mathbb{R}^{+}, \Sigma, \mu\right)$ - пространство измеримых относительно меры $\mu$ функций $x: \mathbb{R}^{+} \rightarrow \mathbb{R}$. Через $S(\mu)^{+}$обозначим конус неотрицательных функций в $S(\mu)$ :

$$
S(\mu)^{+}=\{x \in S(\mu): x(t) \geqslant 0 \text { п.в. }\},
$$

а через $S(\mu)^{c}$ обозначим конус монотонно убываюших функций в $S(\mu)$ :

$$
S(\mu)^{c}=\left\{x \in S(\mu)^{+}: x(t) \downarrow\right\} .
$$

Напомним [4], [10], что пространство $X$ назьвается идеальныцм, если из вьполнения п.в. неравенства $|x(t)| \leqslant|y(t)|$ и из $x \in S(\mu), y \in X$ следует, что $x \in X$ и выполнено неравенство $\|x|X\|\leqslant\| y| X\|$. (Символом $\|x \mid X\|$ обозначается норма элемента $x$ в пространстве $X$.) Для идеального пространства $X$ символами $X^{+}$, $X^{c}$ обозначим конусы в пространстве $X$ :

$$
X^{+}=S(\mu)^{+} \cap X, \quad X^{c}=S(\mu)^{c} \cap X .
$$

Будем считать, что на конусах $X^{+}, X^{c}$ норма индуцирована нормой из $X$, т.е. для $x \in X^{+}$верно равенство $\left\|x\left|X^{+}\|=\| x\right| X\right\|$, а для $x \in X^{c}$ верно равенство $\left\|x\left|X^{c}\|=\| x\right| X\right\|$. Классическими примерами идеальных пространств являются пространства Лебега $L^{p}$, Орлича $L_{N}$, Лоренца $\Lambda(\psi)$, Марцинкевича $M(\psi)$. Изложение теории идеальных пространств можно найти в [4], [10], [11].

Если $X$ - идеальное пространство, $\omega \in S(\mu)^{+}$- весовая функция, то символом $X(\omega)$ обозначается новое идеальное пространство, норма в котором задается равенством

$$
\|x|X(\omega)\|=\| x \omega| X\| .
$$

В частности, в пространстве $L^{p}(\omega)$ норма имеет вид

$$
\left\|x \mid L^{p}(\omega)\right\|=\left(\int_{0}^{\infty}|x(t) \omega(t)|^{p} d \mu(t)\right)^{1 / p}
$$


с обычной модификацией при $p=\infty$.

Введем теперь классы операторов, которые будут играть важную роль в работе.

Будем говорить, что оператор $T$ положителен $(T \in P)$, если он переводит положительные функции в положительные.

Будем говорить, что линейный оператор $T$ принадлежит классу $B(T \in B)$, если он положительный и переводит положительные функции в убывающие.

Класс $B$ достаточно широк. Например, он содержит оператор интегрирования

$$
Q x(t)=\int_{t}^{\infty} x(s) d \mu(s)
$$

и, более общо, операторы Вольтерра

$$
K x(t)=\int_{t}^{\infty} k(t, s) x(s) d \mu(s)
$$

если ядро $k(t, s)$ положительно и убывает по первой переменной. Отметим, что для положительных линейных операторов для всех $x \in S(\mu)^{+}$и $t \in \mathbb{R}^{+}$верно неравенство

$$
|T x(t)| \leqslant T|x|(t) .
$$

Действительно,

$$
\begin{aligned}
& 0 \leqslant T(|x|(t)+x(t))=T|x|(t)+T x(t) \\
& 0 \leqslant T(|x|(t)-x(t))=T|x|(t)-T x(t)
\end{aligned}
$$

поэтому выполнено (1).

Таким образом, если $X, Y$ - идеальные пространства и $T$ - положительный оператор, то из (1) следует равенство

$$
\|T \mid X \rightarrow Y\|=\sup \left(\left\|T x\left|Y^{+}\left\|: x \in X^{+},\right\| x\right| X\right\| \leqslant 1\right) .
$$

(Символом $\|T \mid X \rightarrow Y\|$ обозначается норма оператора $T$ из пространства $X$ в пространство $Y$.) Аналогично, если $T$ - оператор класса $B$, то

$$
\|T \mid X \rightarrow Y\|=\sup \left(\left\|T x\left|Y^{c}\left\|: x \in X^{+},\right\| x\right| X\right\| \leqslant 1\right) .
$$

Напомним теперь некоторые обозначения и факты из теории интерполящии линейных операторов вещественным методом интерполяции [1]-[3].

Пусть $A$ - некоторое линейное топологическое пространство. Пара банаховых пространств $\left(A_{0}, A_{1}\right)$, непрерывно вложенных в пространство $A$, называется $и н$ терполяционной парой. Пусть $\left(A_{0}, A_{1}\right)$ - интерполяционная пара пространств. Для каждого $a \in A_{0}+A_{1}$ и $t>0$ определен функционал Питре

$$
K\left(t, a ; A_{0}, A_{1}\right)=\inf \left(\left\|a_{0}\left|A_{0}\|+t\| a_{1}\right| A_{1}\right\|: a=a_{0}+a_{1}\right) .
$$


При каждом $t$ эти функционалы являются нормой на $A_{0}+A_{1}$. Именно функционал Питре является базой для определения вещественного метода интерполяции. Наряду с классическим $K\left(t, a ; A_{0}, A_{1}\right)$-функционалом для интерполяционной пары идеальных пространств $\left(X_{0}, X_{1}\right)$ на соответствуюших конусах можно определить следующие функционалы:

$$
\begin{aligned}
K^{+}\left(t, x ; X_{0}, X_{1}\right) & =\inf \left(\left\|x_{0}\left|X_{0}\|+t\| x_{1}\right| X_{1}\right\|: x_{0} \in X_{0}^{+}, x_{1} \in X_{1}^{+} ; x=x_{0}+x_{1}\right) \\
K^{c}\left(t, x ; X_{0}, X_{1}\right) & =\inf \left(\left\|x_{0}\left|X_{0}\|+t\| x_{1}\right| X_{1}\right\|: x_{0} \in X_{0}^{c}, x_{1} \in X_{1}^{c} ; x=x_{0}+x_{1}\right) .
\end{aligned}
$$

Поскольку $K^{+}$и $K^{c}$ для каждого $x$ есть нижняя грань линейных функций по $t$, то $K^{+}$и $K^{c}$ как функции $t$ являются вогнутыми.

Отметим, что функционал $K^{+}\left(t, x ; X_{0}, X_{1}\right)$ определен на конусе $X_{0}^{+}+X_{1}^{+}$, а функционал $K^{c}\left(t, x ; X_{0}, X_{1}\right)$ определен на конусе $X_{0}^{c}+X_{1}^{c}$. Легко видеть, что на общей области определения справедливы неравенства

$$
K\left(t, x ; X_{0}, X_{1}\right) \leqslant K^{+}\left(t, x ; X_{0}, X_{1}\right) \leqslant K^{c}\left(t, x ; X_{0}, X_{1}\right) .
$$

В дальнейшем нам потребуется следующее важное неравенство:

$$
K^{+}\left(t, x ; X_{0}, X_{1}\right) \leqslant 2 K\left(t, x ; X_{0}, X_{1}\right)
$$

которое можно легко извлечь из работ [12], [13].

Пусть $\Phi$ - идеальное пространство на $\mathbb{R}^{+}$, содержащее функцию $\min (1, t)$.

Пространство $\left(X_{0}, X_{1}\right)_{K, \Phi}$ состоит из тех $x \in X_{0}+X_{1}$, для которых конечна норма

$$
\left\|x\left|\left(X_{0}, X_{1}\right)_{K, \Phi}\|=\| K\left(t, x ; X_{0}, X_{1}\right)\right| \Phi\right\| .
$$

Это пространство является интерполяционным [1]-[3].

Рассмотрим случай, когда идеальное пространство $\Phi$ есть пространство $\Phi_{\theta, q}$ $(0<\theta<1,1 \leqslant q \leqslant \infty)$, состоящее из тех функций, для которых конечна норма

$$
\left\|x \mid \Phi_{\theta, q}\right\|=\left(\int_{0}^{\infty}\left(t^{-\theta}|x(t)|\right)^{q} \frac{d t}{t}\right)^{1 / q}
$$

со стандартной модификашией при $q=\infty$.

Наряду с пространством $\left(X_{0}, X_{1}\right)_{K, \Phi}$ введем следуюшие множества. Множество $\left(X_{0}, X_{1}\right)_{K^{+}, \Phi}$ состоит из тех $x \in X_{0}^{+}+X_{1}^{+}$, для которых конечен функционал

$$
\left\|x\left|\left(X_{0}, X_{1}\right)_{K^{+}, \Phi}\|=\| K^{+}\left(t, x ; X_{0}, X_{1}\right)\right| \Phi\right\| .
$$

Множество $\left(X_{0}, X_{1}\right)_{K^{c}, \Phi}$ состоит из тех $x \in X_{0}^{c}+X_{1}^{c}$, для которых конечен функционал

$$
\left\|x\left|\left(X_{0}, X_{1}\right)_{K^{c}, \Phi}\|=\| K^{c}\left(t, x ; X_{0}, X_{1}\right)\right| \Phi\right\| .
$$

Соотношения (4) и (5) показывают, что конус положительных функций интер-

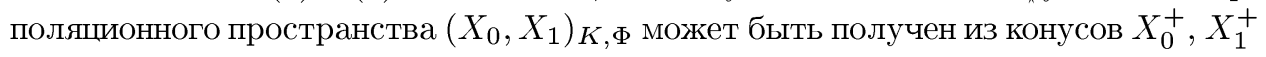


положительных функций пространств $X_{0}$ и $X_{1}$ с помошью аналогичных конструкций.

Оформим это наблюдение в виде леммы.

ЛЕмма 1. Пусть $\left(X_{0}, X_{1}\right)$ - интерполячионная пара идеальных пространств, $\Phi$ - идеальное пространство на $\mathbb{R}^{+}$. Тогда верно равенство

$$
\left(X_{0}, X_{1}\right)_{K, \Phi}^{+}=\left(X_{0}^{+}, X_{1}^{+}\right)_{K, \Phi},
$$

причем для всех $x \in\left(X_{0}, X_{1}\right)_{K, \Phi}^{+}$верны неравенства

$$
\left\|x\left|\left(X_{0}, X_{1}\right)_{K, \Phi}^{+}\|\leqslant\| x\right|\left(X_{0}^{+}, X_{1}^{+}\right)_{K, \Phi}\right\| \leqslant 2\left\|x \mid\left(X_{0}, X_{1}\right)_{K, \Phi}^{+}\right\| .
$$

Используя равенство (2), придем к следующему варианту классической интерполящионной теоремы для вешественного метода интерполяции для положительных операторов.

Теорема 1. Пусть $\left(X_{0}, X_{1}\right),\left(Y_{0}, Y_{1}\right)$ - две интерполяционные пары идеальных пространств. Пусть $T$ - положительный оператор $u\left\|T \mid X_{i} \rightarrow Y_{i}\right\|=M_{i}$ $(i=0,1)$. Тогда

$$
\begin{aligned}
& T:\left(X_{0}^{+}, X_{1}^{+}\right)_{K, \Phi} \rightarrow\left(Y_{0}^{+}, Y_{1}^{+}\right)_{K, \Phi}, \\
& \left\|T \mid\left(X_{0}^{+}, X_{1}^{+}\right)_{K, \Phi} \rightarrow\left(Y_{0}^{+}, Y_{1}^{+}\right)_{K, \Phi}\right\| \leqslant \max \left(M_{0}, M_{1}\right) .
\end{aligned}
$$

Аналог этой теоремы для операторов класса $B$ можно получить так.

Лемма 2. Пусть $\left(X_{0}, X_{1}\right),\left(Y_{0}, Y_{1}\right)$ - две интерполяиионные пары идеальных пространств. Пусть $T$ - оператор класса $B u\left\|T \mid X_{i} \rightarrow Y_{i}\right\|=M_{i}$ $(i=0,1)$. Тогда для любого $x \in X_{0}^{+}+X_{1}^{+}$справедливо неравенство

$$
K^{c}\left(t, T x ; Y_{0}, Y_{1}\right) \leqslant \max \left(M_{0}, M_{1}\right) K\left(t, x ; X_{0}^{+}, X_{1}^{+}\right) .
$$

ДОКАЗАТЕЛЬСтвО. Действительно,

$$
\begin{aligned}
K^{c}\left(t, T x ; Y_{0}, Y_{1}\right) & =\inf \left\{\left\|g_{0}\left|Y_{0}\|+t\| g_{1}\right| Y_{1}\right\|: T x=g_{0}+g_{1} ; g_{0} \in Y_{0}^{c}, g_{1} \in Y_{1}^{c}\right\} \\
& \leqslant \inf \left\{\left\|T x_{0}\left|X_{0}\|+t\| T x_{1}\right| X_{1}\right\|: x=x_{0}+x_{1} ; x_{0} \in X_{0}^{+}, x_{1} \in X_{1}^{+}\right\} \\
& \leqslant \max \left(M_{0}, M_{1}\right) K\left(t, x ; X_{0}^{+}, X_{1}^{+}\right) .
\end{aligned}
$$

Лемма доказана.

Используя леммы 1 и 2 , прийдем к следующей теореме.

Теорема 2. Пусть $\left(X_{0}, X_{1}\right),\left(Y_{0}, Y_{1}\right)$ - две интерполяционные пары идеальных пространств. Пусть $T$ - оператор класса $B$ u $\left\|T \mid X_{i} \rightarrow Y_{i}\right\|=M_{i}$ $(i=0,1)$. Тогда

$$
\begin{aligned}
& T:\left(X_{0}, X_{1}\right)_{K, \Phi}^{+} \rightarrow\left(Y_{0}^{c}, Y_{1}^{c}\right)_{K, \Phi}, \\
& \left\|T \mid\left(X_{0}, X_{1}\right)_{K, \Phi}^{+} \rightarrow\left(Y_{0}^{c}, Y_{1}^{c}\right)_{K, \Phi}\right\| \leqslant 2 \max \left(M_{0}, M_{1}\right) .
\end{aligned}
$$

Именно последняя теорема и является усилением классической интерполяционной теоремы для вещественного метода интерполяции Питре для операторов класса $B$. Это связано с тем, что конус $\left(Y_{0}^{c}, Y_{1}^{c}\right)_{K, \Phi}$ может быть сушественно у́же конуса $\left(Y_{0}^{+}, Y_{1}^{+}\right)_{K, \Phi}$, который согласно лемме 1 совпадает с конусом $\left(Y_{0}, Y_{1}\right)_{K, \Phi}^{+}$. В следуюшем параграфе мы продемонстрируем эту возможность. 


\section{§ 3. Некоторые результаты о вычислении $K^{c}$-функционала}

Пусть $\omega$ - некоторая положительная функция на $\mathbb{R}^{+}$. Положим для всех $t \in \mathbb{R}^{+}$

$$
\widehat{\omega}(t)=\operatorname{ess} \sup \{\omega(s): s \leqslant t\} .
$$

Очевидно, что $\widehat{\omega}(t)$ - положительная возрастающая функция. Отметим, что если п.в. верно равенство $\omega_{0}(t)=\omega_{1}(t)$, то всюду верно равенство $\widehat{\omega}_{0}(t)=\widehat{\omega}_{1}(t)$. Поэтому, если это необходимо, можно исправить $\omega(t)$ на множестве меры нуль так, чтобы вычислять в (6) вместо сушественной верхней грани обычную.

Следуюшая лемма показывает, что при вычислении нормы убывающей функции $x(t)$ в пространстве $L^{\infty}(\omega)$ можно заменить $\omega(t)$ на $\widehat{\omega}(t)$.

ЛЕмма 3. Для любого положительного измеримого веса $\omega(t)$ и функиии $x \in S(\mu)^{c}$ верно равенство

$$
\left\|x\left|L^{\infty}(\omega)\|=\| x\right| L^{\infty}(\widehat{\omega})\right\|
$$

ДоКАЗАТЕЛЬСТВО. Пусть $x \in L^{\infty}(\omega)^{c}$. Без ограничения обшности можно считать, что функции $x(t)$ и $\widehat{\omega}(t)$ непрерывны слева. Так как функция $x$ монотонно убывает и п.в. $\omega(t) \leqslant \widehat{\omega}(t)$, то для п.в. $t$ верно соотношение

$$
x(t) \omega(t) \leqslant x(t) \widehat{\omega}(t) .
$$

Поэтому

$$
\left\|x\left|L^{\infty}(\omega)\|\leqslant\| x\right| L^{\infty}(\widehat{\omega})\right\| .
$$

Докажем обратное неравенство. Пусть

$$
\left\|x \mid L^{\infty}(\omega)\right\|=M<\infty .
$$

Это означает, что п.в. выполнено неравенство

$$
x(t) \leqslant \frac{M}{\omega(t)} .
$$

Поскольку $x(t)$ убывает и непрерьвна слева, то верно соотношение

$$
\begin{aligned}
x(t) & =\operatorname{essinf}\{x(s): s \leqslant t\} \leqslant \operatorname{essinf}\left\{\frac{M}{\omega(s)}: s \leqslant t\right\} \\
& =\frac{M}{\operatorname{ess} \sup \{\omega(s): s \leqslant t\}}=\frac{M}{\widehat{\omega}(t)} .
\end{aligned}
$$

Вспоминая определение нормы в $L^{\infty}(\widehat{\omega})$, окончательно получим

$$
\left\|x\left|L^{\infty}(\widehat{\omega})\|\leqslant\| x\right| L^{\infty}(\omega)\right\| .
$$

Объединяя (8) и (9), придем к (7). Лемма доказана. 
Непосредственно из леммы получим, что для любого $x \in S(\mu)^{c}$ верно равенство

$$
K^{c}\left(t, x ; X, L^{\infty}(\omega)\right)=K^{c}\left(t, x ; X, L^{\infty}(\widehat{\omega})\right)
$$

Используя очевидную формулу

$$
K^{c}\left(t, x ; X_{0}, X_{1}\right)=\frac{1}{t} K^{c}\left(t, x ; X_{1}, X_{0}\right)
$$

проиллюстрируем применение формулы (10) одним примером.

Пусть $\omega_{0}, \omega_{1} \in S(\mu)^{c}, x \in S(\mu)^{c}$ и $c_{0}=\lim _{s \rightarrow+0} \omega_{0}(s), c_{1}=\lim _{s \rightarrow+0} \omega_{1}(s)$. Тогда справедливо равенство

$$
K^{c}\left(t, x ; L^{\infty}\left(\omega_{0}\right), L^{\infty}\left(\omega_{1}\right)\right)=\min \left(c_{0}, c_{1} t\right) \lim _{s \rightarrow+0} x(s)
$$

Действительно,

$$
\begin{aligned}
K^{c}\left(t, x ; L^{\infty}\left(\omega_{0}\right), L^{\infty}\left(\omega_{1}\right)\right) & =K^{c}\left(t, x ; L^{\infty}\left(\widehat{\omega}_{0}\right), L^{\infty}\left(\widehat{\omega}_{1}\right)\right) \\
& =K^{c}\left(t, x ; L^{\infty}\left(c_{0}\right), L^{\infty}\left(c_{1}\right)\right)=x(+0) \min \left(c_{0}, c_{1} t\right)
\end{aligned}
$$

Прежде чем переходить к основной теореме этого параграфа, введем два понятия.

Пусть $x(t), \omega(t) \in S(\mu)^{+}$. Для каждого $t \in \mathbb{R}^{+}, \alpha \in \mathbb{R}^{+}$положим

$$
x_{\alpha, \omega}(t)=\max \left\{x(t)-\frac{\alpha}{\omega(t)}, 0\right\}
$$

Пусть $x \in S(\mu)^{+}$. Для каждого $t$ положим

$$
\tilde{x}(t)=\operatorname{ess} \sup \{x(s): s \geqslant t\}
$$

Очевидно, что функция $\tilde{x}(t)$ убывает. Если это необходимо, то можно исправить $x(t)$ на множестве меры нуль так, чтобы вычислять в (12) вместо существенной верхней грани обычную.

Теперь мы можем сформулировать основную теорему.

ТЕОРема 3. Пусть $X$ - идеальное пространство и $\omega(t)$ - положительный измеримый вес. Тогда для любой функции $x(t) \in S(\mu)^{c}$ верно равенство

$$
K^{c}\left(t, x ; X, L^{\infty}(\omega)\right)=\inf \left\{\left\|\tilde{x}_{\alpha, \widehat{\omega}} \mid X\right\|+t \alpha: \alpha \in \mathbb{R}^{+}\right\}
$$

(Функция $\tilde{x}_{\alpha, \widehat{\omega}}$ определена с помошью формул (6), (11), (12); сначала строим функцию $x_{\alpha, \widehat{\omega}}$, а затем функцию $\tilde{x}_{\alpha, \widehat{\omega}}$.) 
ДокАЗАТЕЛЬСтво. Согласно лемме 3 верно равенство

$$
\left\|x\left|L^{\infty}(\omega)\|=\| x\right| L^{\infty}(\widehat{\omega})\right\| .
$$

Поэтому достаточно рассмотреть случай, когда $\omega(t)$ - положительная возрастающая функция и, следовательно, п.в. выполнено равенство $\omega(t)=\widehat{\omega}(t)$.

Докажем сначала неравенство

$$
K^{c}\left(t, x ; X, L^{\infty}(\omega)\right) \leqslant \inf \left\{\left\|\tilde{x}_{\alpha, \widehat{\omega}} \mid X\right\|+t \alpha: \alpha \in \mathbb{R}^{+}\right\}
$$

Без ограничения общности можно считать, что функции $x(t)$ и $\omega(t)$ непрерывны справа. Тогда функция $x_{\alpha, \omega}$ также непрерывна справа. Из того что п.в. выполнено равенство $\omega(t)=\widehat{\omega}(t)$, следует справедливость соотношения

$$
\begin{aligned}
\tilde{x}_{\alpha, \omega}(t) & =\operatorname{ess} \sup \left\{x_{\alpha, \omega}(s): s \geqslant t\right\}=\operatorname{ess} \sup \left\{\max \left\{x(s)-\frac{\alpha}{\omega(s)}, 0\right\}: s \geqslant t\right\} \\
& =\max \left\{\operatorname{ess} \sup \left\{x(s)-\frac{\alpha}{\omega(s)}: s \geqslant t\right\}, 0\right\} \\
& =\max \left\{\operatorname{ess} \sup \left\{x(s)-\frac{\alpha}{\widehat{\omega}(s)}: s \geqslant t\right\}, 0\right\} \\
& =\operatorname{ess} \sup \left\{\max \left\{x(s)-\frac{\alpha}{\widehat{\omega}(s)}, 0\right\}: s \geqslant t\right\}=\tilde{x}_{\alpha, \widehat{\omega}}(t)
\end{aligned}
$$

Из неравенства

$$
x(t) \geqslant \sup \{x(s): s \geqslant t\} \geqslant \sup \left\{\max \left\{x(s)-\frac{\alpha}{\omega(s)}, 0\right\}: s \geqslant t\right\}=\tilde{x}_{\alpha, \omega}(t)
$$

следует, что функция $x_{0}(t)=x(t)-\tilde{x}_{\alpha, \omega}(t)$ неотрицательна. Проверим, что она убывает на $\mathbb{R}^{+}$. Пусть $t_{0}<t_{1}$. Если $\tilde{x}_{\alpha, \omega}\left(t_{0}\right)=\tilde{x}_{\alpha, \omega}\left(t_{1}\right)$, то

$$
x_{0}\left(t_{0}\right)-x_{0}\left(t_{1}\right)=x\left(t_{0}\right)-x\left(t_{1}\right) \geqslant 0 .
$$

Если же $\tilde{x}_{\alpha, \omega}\left(t_{0}\right)>\tilde{x}_{\alpha, \omega}\left(t_{1}\right)$, то

$$
\tilde{x}_{\alpha, \omega}\left(t_{0}\right)=\sup \left\{x(s)-\frac{\alpha}{\omega(s)}: t_{0} \leqslant s<t_{1}\right\} .
$$

Для каждого $n$ выберем $s(n) \in\left[t_{0}, t_{1}\right)$ так, чтобы

$$
\tilde{x}_{\alpha, \omega}\left(t_{0}\right) \leqslant x(s(n))-\frac{\alpha}{\omega(s(n))}+\frac{1}{n} .
$$

Тогда из неравенства

$$
x_{0}\left(t_{1}\right)=x\left(t_{1}\right)-\sup \left\{x(s)-\frac{\alpha}{\omega(s)}: s \geqslant t_{1}\right\} \leqslant \frac{\alpha}{\omega\left(t_{1}\right)},
$$


неравенства (17) и убывания функций $x(t)$ и $\frac{1}{\omega(t)}$ получим

$$
x_{0}\left(t_{0}\right)-x_{0}\left(t_{1}\right)>x\left(t_{0}\right)-x(s(n))+\frac{\alpha}{\omega(s(n))}-\frac{\alpha}{\omega\left(t_{1}\right)}-\frac{1}{n} \geqslant-\frac{1}{n} .
$$

Из (18) с помошью предельного перехода получим, что и в этом случае тоже выполняется неравенство (16). Таким образом, мы убедились, что $x_{0}$ убывает на $\mathbb{R}^{+}$.

Оценим теперь величину $\left\|x-\tilde{x}_{\alpha, \omega} \mid L^{\infty}(\omega)\right\|$.

Из убывания функции $x(t)$ и определения функции $\tilde{x}_{\alpha, \omega}(t)$ получаем соотношение

$$
\begin{aligned}
\left|x(t)-\tilde{x}_{\alpha, \omega}(t)\right| & =x(t)-\tilde{x}_{\alpha, \omega}(t)=x(t)-\sup \left\{x(s)-\frac{\alpha}{\omega(s)}: s \geqslant t\right\} \\
& \leqslant x(t)-\left(x(t)-\frac{\alpha}{\omega(t)}\right)=\frac{\alpha}{\omega(t)} .
\end{aligned}
$$

Поэтому

$$
\left\|x-\tilde{x}_{\alpha, \omega} \mid L^{\infty}(\omega)\right\| \leqslant \alpha .
$$

Если учесть выполнение п.в. соотношения (15), то можно выписать оценку сверху для $K^{c}$-функционала:

$$
K^{c}\left(t, x ; X, L^{\infty}(\omega)\right) \leqslant \inf \left\{\left\|\tilde{x}_{\alpha, \widehat{\omega}} \mid X\right\|+t \alpha: \alpha \in \mathbb{R}^{+}\right\}
$$

Таким образом, (14) доказано.

Докажем теперь обратное неравенство:

$$
K^{c}\left(t, x ; X, L^{\infty}(\omega)\right) \geqslant \inf \left\{\left\|\tilde{x}_{\alpha, \widehat{\omega}} \mid X\right\|+t \alpha: \alpha \in \mathbb{R}^{+}\right\} .
$$

Пусть $g_{0}, g_{1} \in S(\mu)^{c}$ и почти при всех $t$ верно равенство $x(t)=g_{0}(t)+g_{1}(t)$. Если положить

$$
\left\|g_{1}\left|L^{\infty}(\omega)\|=\| g_{1}\right| L^{\infty}(\widehat{\omega})\right\|=\alpha,
$$

то п.в. выполнено неравенство

$$
0 \leqslant g_{1}(t) \leqslant \frac{\alpha}{\widehat{\omega}(t)} .
$$

Поэтому из соотношения

$$
g_{0}(t)=x(t)-g_{1}(t) \geqslant x(t)-\frac{\alpha}{\widehat{\omega}(t)}
$$

и неравенства $g_{0}(t) \geqslant 0$ получим, что п.в. выполнено неравенство

$$
g_{0}(t) \geqslant \max \left\{0, x(t)-\frac{\alpha}{\widehat{\omega}(t)}\right\} .
$$


Без ограничения общности можно считать, что $g_{0}$ непрерывна справа. Так как $g_{0}$ убывает, то для всех $t$ получим

$$
g_{0}(t) \geqslant \sup \left\{g_{0}(s): s \geqslant t\right\} \geqslant \sup \left\{\max \left\{x(s)-\frac{\alpha}{\widehat{\omega}(s)}, 0\right\}: s \geqslant t\right\}=\tilde{x}_{\alpha, \widehat{\omega}}(t)
$$

Из (20), (21) и идеальности пространства $X$ получим, что

$$
K^{c}\left(t, x ; X, L^{\infty}(\omega)\right) \geqslant \inf \left\{\left\|\tilde{x}_{\alpha, \widehat{\omega}} \mid X\right\|+t \alpha: \alpha \in \mathbb{R}^{+}\right\}
$$

Объединяя (14) и (19), придем к (13). Теорема доказана.

Отметим, что формула (13) очень напоминает известную формулу для вычисления $K$-функционала Питре через $E$-фуункционал [1], [3].

Приведем теперь пример вычисления $K^{c}$-функционала для конкретной пары идеальных пространств.

ТЕОРема 4. Пусть $u \in S(\mu)^{+}$- некоторый вес, для которого при некотором $s>0$ верно соотношение

$$
\int_{0}^{s} u(\tau) d \mu(\tau)<\infty
$$

Положсим

$$
\int_{0}^{\infty} u(\tau) d \mu(\tau)=M
$$

Пусть $x \in S(\mu)^{c}$. Для кажсдого $t \in(0, M)$ определим число $\tau(t)$ из уравнения

$$
\int_{0}^{\tau(t)} u(s) d \mu(s)=t
$$

Тогда при $t \in(0, M)$ справедливо равенство

$$
K^{c}\left(t, x ; L^{1}(u), L^{\infty}\right)=\int_{0}^{\tau(t)} x(s) u(s) d \mu(s)
$$

Eсли же $t \geqslant M$, mo

$$
K^{c}\left(t, x ; L^{1}(u), L^{\infty}\right)=\int_{0}^{\infty} x(s) u(s) d \mu(s)
$$


ДокАЗАТЕЛЬСТво. Пусть $x \in S(\mu)^{c}$. Без ограничения общности можно считать, что $x(t)$ непрерывна справа. Поскольку $\omega(t) \equiv 1$, то функция $x_{\alpha, 1}$ убывает, и поэтому справедливо равенство $\tilde{x}_{\alpha, 1} \equiv x_{\alpha, 1}$. Поэтому, применяя предыдушую теорему, прийдем к формуле

$$
\begin{aligned}
K^{c}\left(t, x ; L^{1}(u), L^{\infty}\right) & =\inf \left\{\left\|\tilde{x}_{\alpha, 1} \mid L^{1}(u)\right\|+t \alpha: \alpha \in \mathbb{R}^{+}\right\} \\
& =\inf \left\{\left\|x_{\alpha, 1} \mid L^{1}(u)\right\|+t \alpha: \alpha \in \mathbb{R}^{+}\right\}
\end{aligned}
$$

Разберем сначала случай $t \in(0, M)$. Положим

$$
G(x, \alpha)=\left\|x_{\alpha, 1} \mid L^{1}(u)\right\|+t \alpha .
$$

Покажем сначала, что для любого $\alpha$ справедливо неравенство

$$
G(x, \alpha) \geqslant \int_{0}^{\tau(t)} x(s) u(s) d \mu(s) .
$$

Это можно сделать так. Из непрерывности справа и монотонности функции $x$ следует, что

$$
J(\alpha)=\left\{s: x_{\alpha, 1}(s)>0\right\}=\left[0, \tau^{*}(\alpha)\right) .
$$

Если $\tau^{*}(\alpha)<\tau(t)$, то $x(s)<\alpha$ при $s \in\left(\tau^{*}(\alpha), \tau(t)\right)$, и поэтому

$$
\begin{aligned}
G(x, \alpha) & =\int_{0}^{\tau^{*}(\alpha)} x(s) u(s) d \mu(s)-\alpha \int_{0}^{\tau^{*}(\alpha)} u(s) d \mu(s)+\alpha \int_{0}^{\tau(t)} u(s) d \mu(s) \\
& =\int_{0}^{\tau^{*}(\alpha)} x(s) u(s) d \mu(s)+\alpha \int_{\tau^{*}(\alpha)}^{\tau(t)} u(s) d \mu(s) \\
& \geqslant \int_{0}^{\tau(t)} x(s) u(s) d \mu(s)
\end{aligned}
$$

Если $\tau^{*}(\alpha)>\tau(t)$, то $x(s)>\alpha$ при $s \in\left[0, \tau^{*}(\alpha)\right)$, и поэтому

$$
\begin{aligned}
G(x, \alpha) & =\int_{0}^{\tau^{*}(\alpha)} x(s) u(s) d \mu(s)-\alpha \int_{0}^{\tau^{*}(\alpha)} u(s) d \mu(s)+\alpha \int_{0}^{\tau(t)} u(s) d \mu(s) \\
& =\int_{0}^{\tau(t)} x(s) u(s) d \mu(s)+\int_{\tau(t)}^{\tau^{*}(\alpha)} x(s) u(s) d \mu(s)-\alpha \int_{\tau(t)}^{\tau^{*}(\alpha)} u(s) d \mu(s) \\
& \geqslant \int_{0}^{\tau(t)} x(s) u(s) d \mu(s) .
\end{aligned}
$$

Таким образом, из (25) и (26) следует, что неравенство (24) выполнено, и поэтомy

$$
K^{c}\left(t, x ; L^{1}(u), L^{\infty}\right) \geqslant \int_{0}^{\tau(t)} x(s) u(s) d \mu(s) .
$$


Докажем теперь обратное неравенство

$$
K^{c}\left(t, x ; L^{1}(u), L^{\infty}\right)=\inf \left\{G(x, \alpha): \alpha \in \mathbb{R}^{+}\right\} \leqslant \int_{0}^{\tau(t)} x(s) u(s) d \mu(s) .
$$

Выберем непрерывную функцию $g(t)$, которая удовлетворяет условиям: для всех $t \in \mathbb{R}^{+}$верно неравенство $g(t)>0$; если $s>t$, то $g(s)<g(t)$ и

$$
\int_{0}^{\infty} g(s) d \mu(s)=C<\infty
$$

Для каждого $n \in \mathbb{N}$ положим

$$
x_{n}(t)= \begin{cases}x(t)+\frac{g(t)}{n} & \text { при } t<\tau(t), \\ x(t) & \text { при } t \geqslant \tau(t) .\end{cases}
$$

Тогда $x_{n}(t) \geqslant x(t)$ всюду и

$$
\left\{s: x_{n}(s)>x(\tau(t))\right\}=[0, \tau(t)) .
$$

Поэтому

$$
\begin{aligned}
\inf \left\{G(x, \alpha): \alpha \in \mathbb{R}^{+}\right\} \leqslant & \inf \left\{G\left(x_{n}, \alpha\right): \alpha \in \mathbb{R}^{+}\right\} \leqslant G\left(x_{n}, x(\tau(t))\right) \\
= & \int_{0}^{\tau(t)} x_{n}(s) u(s) d \mu(s)-x(\tau(t)) \int_{0}^{\tau(t)} u(s) d \mu(s) \\
& +x(\tau(t)) \int_{0}^{\tau(t)} u(s) d \mu(s) \\
\leqslant & \int_{0}^{\tau(t)} x(s) u(s) d \mu(s)+\frac{c}{n} .
\end{aligned}
$$

Устремляя в (29) $n$ к бесконечности, придем к (28). Объединяя (27) и (28), получим (23).

Случай $t \geqslant M$ разбирается аналогично. Теорема доказана.

Отметим один частньй случай теоремы 4 . Пусть $u(t) \equiv 1$ и $\mu(s)$ есть мера Лебега на $\mathbb{R}^{+}$. Тогда из теоремы 4 получим, что для всех $x \in S(\mu)$ верно равенство

$$
K^{c}\left(t, x ; L^{1}, L^{\infty}\right)=\int_{0}^{t} x(s) d s .
$$

Если заметить, что для $x \in S(\mu)^{c}$ справедливо равенство $x(t) \equiv x^{*}(t)$ (напомним, что для функции $x$ перестановка $x^{*}(t)$ по убыванию определяется равенством $\left.x^{*}(t)=\inf \{\tau: \mu(s:|x(s)| \geqslant \tau)<t\}\right)$, то (30) совпадает на $S(\mu)^{c}$ с известной формулой Питре [1]-[5] для $K$-функционала пары $\left(L^{1}, L^{\infty}\right)$.

Результаты, аналогичные теоремам 3,4 , справедливы и для $K^{+}$-функционала. Сформулируем их для полноты изложения. 
ТЕОРема 5. Пусть $X$ - идеальное пространство и $\omega(t)$ - положительный измеримый вес. Тогда для любой функиии $x(t) \in S(\mu)^{+}$верно равенство

$$
K^{+}\left(t, x ; X, L^{\infty}(\omega)\right)=\inf \left\{\left\|x_{\alpha, \omega} \mid X\right\|+t \alpha: \alpha \in \mathbb{R}^{+}\right\},
$$

где функиия $x_{\alpha, \omega}$ определена равенством (11).

ТЕОРема 6. Пусть $u \in S(\mu)^{+}-$некоторый вес, для которого при некотором $s>0$ верно соотношение

$$
\int_{0}^{s} u(\tau) d \mu(\tau)<\infty
$$

Положим

$$
\int_{0}^{\infty} u(\tau) d \mu(\tau)=M
$$

Пусть $x \in S(\mu)^{+}$. Для каждого $t \in(0, M)$ определим число $\tau(t)$ из уравнения

$$
\int_{0}^{\tau(t)} u(s) d \mu(s)=t
$$

Тогда при $t \in(0, M)$ справедливо равенство

$$
K^{+}\left(t, x ; L^{1}(u), L^{\infty}\right)=\int_{0}^{\tau(t)} x(s) u(s) d \mu(s)
$$

Если же $t \geqslant M$, mо

$$
K^{+}\left(t, x ; L^{1}(u), L^{\infty}\right)=\int_{0}^{\infty} x(s) u(s) d \mu(s)
$$

Формула (30) показывает, что для всех $x \in S(\mu)^{c}$, где $\mu$-мера Лебега, верно равенство

$$
K^{c}\left(t, x ; L^{1}, L^{\infty}\right)=K\left(t, x ; L^{1}, L^{\infty}\right)
$$

Покажем, что в случае весовых пространств между $K^{c}$-функционалом и $K$-функционалом даже может не быть эквивалентности.

Рассмотрим пару $\left(L^{1}, L^{\infty}(\omega)\right)$ на $\mathbb{R}^{+}$, и пусть $\mu$ есть мера Лебега. Пусть вес $\omega$ удовлетворяет следуюшим условиям: $\omega(t)$ - непрерывная функция; $\omega(t)$ убывает; $\omega(0)=1$

$$
\sup \{s \omega(s): s \geqslant 1\}=C<\infty .
$$

Отметим, что из убывания $\omega$ следует, что $\widehat{\omega}(s) \equiv 1$. Тогда для функции $x_{\alpha}(s)=s^{\alpha}$, $\alpha \in(-1,0]$, согласно формулам (10) и (31) будем иметь

$$
K^{c}\left(t, x_{\alpha} ; L^{1}, L^{\infty}(\omega)\right)=K\left(t, x_{\alpha} ; L^{1}, L^{\infty}\right)=\int_{0}^{t} x_{\alpha}(s) d s=\frac{t^{\alpha+1}}{\alpha+1} .
$$


С другой стороны,

$$
K\left(t, x_{\alpha} ; L^{1}, L^{\infty}(\omega)\right) \leqslant \inf \left\{\left\|\chi(D) x_{\alpha}\left|L^{1}\|+t\| \chi\left(\mathbb{R}^{+} \backslash D\right) x_{\alpha}\right| L^{\infty}\right\|: D \subset \mathbb{R}^{+}\right\} .
$$

(Здесь $\chi(D)$ - характеристическая функция множества $D$.) Поэтому

$$
\begin{aligned}
K\left(t, x_{\alpha} ; L^{1}, L^{\infty}(\omega)\right) & \leqslant \inf \left\{\int_{0}^{\tau} x_{\alpha}(s) d s+t \omega(\tau) x_{\alpha}(\tau): \tau \geqslant 0\right\} \\
& =\inf \left\{\frac{\tau^{1+\alpha}}{\alpha+1}+t \omega(\tau) x_{\alpha}(\tau): \tau \geqslant 0\right\} .
\end{aligned}
$$

Для $t>1$ положим $\tau=\sqrt{t}$. Тогда

$$
\begin{aligned}
K\left(t, x_{\alpha} ; L^{1}, L^{\infty}(\omega)\right) & \leqslant \frac{t^{(1+\alpha) / 2}}{\alpha+1}+t \omega(\sqrt{t}) t^{\alpha / 2} \\
& =\frac{t^{(1+\alpha) / 2}}{\alpha+1}+\omega(\sqrt{t}) t^{1+\alpha / 2} .
\end{aligned}
$$

Таким образом, верно неравенство

$$
K\left(t, x_{\alpha} ; L^{1}, L^{\infty}(\omega)\right) \leqslant t^{(\alpha+1) / 2}\left(\frac{1}{1+\alpha}+\omega(\sqrt{t}) \sqrt{t}\right) .
$$

Из условия (32) следует, что при $t \geqslant 1$ верно неравенство

$$
K\left(t, x_{\alpha} ; L^{1}, L^{\infty}(\omega)\right) \leqslant C_{1} t^{(\alpha+1) / 2} .
$$

Поэтому из (33) и (34) получим

$$
\lim _{t \rightarrow \infty} \frac{K\left(t, x_{\alpha} ; L^{1}, L^{\infty}(\omega)\right)}{K^{c}\left(t, x_{\alpha} ; L^{1}, L^{\infty}(\omega)\right)}=0 .
$$

Последнее равенство означает, что $K$-функционал Питре и $K^{c}$-функционал для пары $\left(L^{1}, L^{\infty}(\omega)\right)$ не эквивалентны.

Продемонстрируем сейчас еще один эффект, связанный с различным поведением $K$ - и $K^{c}$-функционалов. А именно мы покажем, что для любого симметричного пространства $X$ найдется пара весов $w_{0}$ и $w_{1}$ такая, что на общем конусе множества

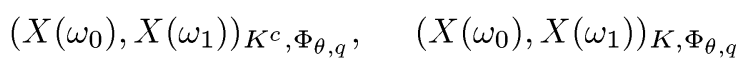

имеют неэквивалентные нормы.

Пусть $X$-идеальное пространство, $\omega_{0}, \omega_{1}$ - две весовые функции, $X\left(\omega_{0}\right), X\left(\omega_{1}\right)$ два весовых идеальных пространства, построенных по одному и тому же $X$. Хорошо известно, что справедлива формула [14, с. 53]

$$
\frac{1}{c}\left\|\min \left(\omega_{0}, t \omega_{1}\right) x\left|X\left\|\leqslant K\left(t, x ; X\left(\omega_{0}\right), X\left(\omega_{1}\right)\right) \leqslant c\right\| \min \left(\omega_{0}, t \omega_{1}\right) x\right| X\right\|,
$$


причем константа $c$ не зависит от $x \in X$ и весов $\omega_{0}, \omega_{1}$.

Пусть $X$ - симметричное пространство. Напомним (см., например, [4]), что идеальное пространство $X$ назьвается симметричным. если из $x \in S(\mu), y \in X$ и выполнения при всех $\alpha \in \mathbb{R}^{+}$неравенства $\lambda(x, \alpha) \leqslant \lambda(y, \alpha)$, где $\lambda(z, \alpha)$ есть функция распределения функции $z: \quad \lambda(z, \alpha)=\mu\{\tau:|z(\tau)|>\alpha\}$, следует, что $x \in X$ и $\|x|X\|\leqslant\| y| X\|$. Многочисленные примеры симметричных пространств приведены в [4].

Далее мы ограничимся случаем, когда областью определения функций из симметричного пространства $X$ и соответственно из $X\left(\omega_{0}\right)$ и $X\left(\omega_{1}\right)$ является множество $(0,1]$, а мера $\mu$ есть мера Лебега. Этого достаточно для демонстрации обещанного нами эффекта. В других случаях рассмотрения аналогичны.

Пусть $a_{i}$ - убывающая положительная последовательность, причем $a_{0}=1$ и

$$
\lim _{i \rightarrow \infty} a_{i}=0
$$

Положим $J_{2 k}=\left(2^{-k}-2^{-(k+2)}, 2^{-k}\right], \quad J_{2 k+1}=\left(2^{-(k+1)}, 2^{-k}-2^{-(k+2)}\right]$, $k=0,1,2, \ldots$ Тогда $\left|J_{2 k}\right|=\left|J_{2 k+1}\right|=2^{-(k+2)}$ и $(0,1]=\bigcup_{i=0}^{\infty} J_{i}$.

Определим веса $\omega_{0}(s), \omega_{1}(s)$ соотношениями

$$
\begin{aligned}
& \omega_{0}(s)=\left\{\begin{array}{lll}
1 & \text { при } & s \in J_{2 i}, \\
a_{i} & \text { при } & s \in J_{2 i+1},
\end{array}\right. \\
& \omega_{1}(s)=\left\{\begin{array}{lll}
a_{i} & \text { при } & s \in J_{2 i}, \\
1 & \text { при } & s \in J_{2 i+1} .
\end{array}\right.
\end{aligned}
$$

Для каждого $x \in S(\mu)^{+}$положим

$$
x_{k}(\tau)=x(\tau) \chi\left(\bigcup_{i=k}^{\infty} J_{2 i}\right)
$$

Покажем сначала, что для $x \in S(\mu)^{c}$ при всех $k$ выполнены неравенства

$$
\min (1, t)\left\|x\left|X\left\|\geqslant K^{c}\left(t, x ; X\left(\omega_{0}\right), X\left(\omega_{1}\right)\right) \geqslant \min (1, t)\right\| x_{k}\right| X\right\|
$$

Следует обратить внимание, что в (36) весовые пространства присутствуют только в центральной части неравенств.

Проверим первое неравенство в (36). Из того, что для $i=0,1$ при всех $\tau$ верно неравенство $0<\omega_{i}(\tau) \leqslant 1$, следует соотношение

$$
K^{c}\left(t, x ; X\left(\omega_{0}\right), X\left(\omega_{1}\right)\right) \leqslant K^{c}(t, x ; X(1), X(1)) \leqslant \min (1, t)\|x \mid X\| .
$$

Поэтому первое неравенство в (36) доказано. 
Проверим второе неравенство в (36). Пусть $x, g_{0}, g_{1} \in S(\mu)^{c}$ и $x(\tau) \equiv g_{0}(\tau)+$ $g_{1}(\tau)$. Положим $y_{0}(\tau)=g_{0}(\tau) \chi\left(\bigcup_{i=k}^{\infty} J_{2 i}\right)$. Тогда из того, что $X-$ идеальное пространство, очевидным образом выполнено неравенство

$$
\begin{aligned}
\left\|g_{0} \mid X\left(\omega_{0}\right)\right\| & \geqslant\left\|g_{0} \chi\left(\bigcup_{i=k}^{\infty} J_{2 i}\right)\left|X\left(\omega_{0}\right)\|=\| \omega_{0} g_{0} \chi\left(\bigcup_{i=k}^{\infty} J_{2 i}\right)\right| X\right\| \\
& =\left\|g_{0} \chi\left(\bigcup_{i=k}^{\infty} J_{2 i}\right)\left|X\|=\| y_{0}\right| X\right\| .
\end{aligned}
$$

Для каждого $i \geqslant k$ и для $\tau \in J_{2 i}$ определим функцию

$$
y_{1}(\tau)=g_{1}\left(\tau-2^{-(i+2)}\right)
$$

и продолжим ее в остальных точках $(0,1]$ нулем. Тогда из включения $g_{1} \in S(\mu)^{c}$ для любого $\tau \in J_{2 i}$ следует неравенство

$$
x(\tau)=g_{0}(\tau)+g_{1}(\tau) \leqslant g_{0}(\tau)+g_{1}\left(\tau-2^{-(i+2)}\right)=y_{0}(\tau)+y_{1}(\tau) .
$$

Поэтому при всех $\tau \in(0,1]$ верно неравенство

$$
x_{k}(\tau) \leqslant y_{0}(\tau)+y_{1}(\tau) .
$$

Кроме того, из симметричности пространства $X$ следует неравенство

$$
\begin{aligned}
\left\|g_{1} \mid X\left(\omega_{1}\right)\right\| & \geqslant\left\|g_{1} \chi\left(\bigcup_{i=k}^{\infty} J_{2 i+1}\right)\left|X\left(\omega_{1}\right)\|=\| \omega_{1} g_{1} \chi\left(\bigcup_{i=k}^{\infty} J_{2 i+1}\right)\right| X\right\| \\
& =\left\|g_{1} \chi\left(\bigcup_{i=k}^{\infty} J_{2 i+1}\right)\left|X\|=\| y_{1}\right| X\right\| .
\end{aligned}
$$

Поэтому из (37)-(39) получим соотношение

$$
\left\|g_{0}\left|X\left(\omega_{0}\right)\|+t\| g_{1}\right| X\left(\omega_{1}\right)\right\| \geqslant\left\|y_{0}\left|X\left(\omega_{0}\right)\|+t\| y_{1}\right| X\left(\omega_{1}\right)\right\| \geqslant \min (1, t)\left\|x_{k} \mid X\right\|
$$

и, следовательно,

$$
K^{c}\left(t, x ; X\left(\omega_{0}\right), X\left(\omega_{1}\right)\right) \geqslant \min (1, t)\left\|x_{k} \mid X\right\| .
$$

Таким образом, (36) полностью доказано.

Покажем теперь, что если $x \in S(\mu)^{c}$ и $\operatorname{supp} x=\chi\left(\bigcup_{i=2 k}^{\infty} J_{i}\right)$, то верны неравенства

$$
\left\|x_{k}|X\|\leqslant\| x| X\right\| \leqslant 3\left\|x_{k} \mid X\right\| .
$$

Отметим, что первое неравенство в (40) следует из идеальности $X$.

Для проверки второго неравенства в (40) можно поступить так. 
Для $\tau \in\left(2^{-(i+1)}, 2^{-(i+1)}+2^{-(i+3)}\right.$ положим $y(\tau)=x_{k}\left(\tau-2^{-(i+3)}\right)$, а для $\tau \in\left(2^{-(i+1)}+2^{-(i+3)}, 2^{-(i+1)}+2^{-(i+2)}\right]$ положим $z(\tau)=x_{k}\left(\tau-2 * 2^{-(i+3)}\right)$, $i=0,1,2, \ldots$, a при остальных $\tau$ доопеделим $y(\tau)$ и $z(\tau)$ нулем.

Тогда из графика $x(\tau)$, включения $x \in S(\mu)^{c}$ и определения $x_{k}$ следует неравенство

$$
x(\tau) \leqslant x_{k}(\tau)+y(\tau)+z(\tau) .
$$

Кроме того, из построения $y(\tau), z(\tau)$ и симметричности пространства $X$ следуют неравенства

$$
\left\|y\left|X\|\leqslant\| x_{k}\right| X\right\|, \quad\left\|z\left|X\|\leqslant\| x_{k}\right| X\right\|
$$

Поэтому и второе неравенство в (40) верно.

С другой стороны,

$$
\min \left(\omega_{0}, t \omega_{1}\right)=\left\{\begin{array}{lll}
\min \left(1, t a_{i}\right) & \text { при } & s \in J_{2 i} \\
\min \left(a_{i}, t\right) & \text { при } & s \in J_{2 i+1}
\end{array}\right.
$$

Поэтому если $i \geqslant k$, то из монотонности $a_{i}$ и (41) получим неравенство

$$
\min \left(\omega_{0}, t \omega_{1}\right) \leqslant \max \left\{\min \left\{a_{k}, t\right\}, \min \left\{1, t a_{k}\right\}\right\}
$$

Непосредственно из (42) следует, что:

- если $0<t<a_{k}$, то $\min \left(\omega_{0}, t \omega_{1}\right) \leqslant \max \left(t, t a_{k}\right) \leqslant t$;

- если $a_{k} \leqslant t \leqslant 1$, то $\min \left(\omega_{0}, t \omega_{1}\right) \leqslant \max \left(a_{k}, t a_{k}\right) \leqslant a_{k}$;

- если $1 \leqslant t \leqslant \frac{1}{a_{k}}$, то $\min \left(\omega_{0}, t \omega_{1}\right) \leqslant \max \left(a_{k}, t a_{k}\right) \leqslant t a_{k}$;

- если $t \geqslant \frac{1}{a_{k}}$, то $\min \left(\omega_{0}, t \omega_{1}\right) \leqslant \max \left(1, a_{k}\right) \leqslant 1$.

Поэтому, используя (35), для $x \in S(\mu)^{c}$ с $\operatorname{supp} x \subset \bigcup_{j=2 k}^{\infty} J_{j}$ получим неравенство

$$
K\left(t, x ; X\left(\omega_{0}\right), X\left(\omega_{1}\right)\right) \leqslant C \begin{cases}t\|x \mid X\| & \text { при } t \in\left(0, a_{k}\right] \\ a_{k}\|x \mid X\| & \text { при } t \in\left(a_{k}, 1\right] \\ t a_{k}\|x \mid X\| & \text { при } t \in\left(1,1 / a_{k}\right] \\ \|x \mid X\| & \text { при } t \in\left(1 / a_{k}, \infty\right) .\end{cases}
$$

Пусть теперь $\varphi_{0}(s), \varphi_{1}(s)$ - две произвольные функции $\varphi_{i}:(0,1) \rightarrow \mathbb{R}^{+}$, удовлетворяюшие условиям: $\varphi_{0}(s)$ возрастает, $\varphi_{1}(s)$ убывает; для всех $s \in(0,1)$ верны неравенства $\varphi_{1}(s) \geqslant 1, \varphi_{0}(s) \leqslant 1$;

$$
\lim _{s \rightarrow 0} \varphi_{1}(s)=\infty, \quad \lim _{s \rightarrow 0} \varphi_{0}(s)=0, \quad \lim _{s \rightarrow 0} s \varphi_{1}(s)=0, \quad \lim _{s \rightarrow 0} \frac{s}{\varphi_{0}(s)}=0
$$


Тогда из (36), (40), (43) и (44) следует соотношение

$$
\begin{aligned}
& \lim _{k \rightarrow \infty} \sup \left\{\frac{K\left(t, x \chi\left(\bigcup_{j=2 k}^{\infty} J_{j}\right) ; X\left(\omega_{0}\right), X\left(\omega_{1}\right)\right)}{K^{c}\left(t, x \chi\left(\bigcup_{j=2 k}^{\infty} J_{j}\right) ; X\left(\omega_{0}\right), X\left(\omega_{1}\right)\right)}:\right. \\
& \left.x \in X^{c}\left(\omega_{0}\right)+X^{c}\left(\omega_{1}\right), t \in\left[\varphi_{0}\left(a_{k}\right), \varphi_{1}\left(a_{k}\right)\right)\right\} \\
& \leqslant 3 C \max \left\{\lim _{k \rightarrow \infty} \sup \left\{\frac{a_{k}\|x \mid X\|}{t\|x \mid X\|}: x \in X^{c}\left(\omega_{0}\right)+X^{c}\left(\omega_{1}\right), t \in\left[\varphi_{0}\left(a_{k}\right), 1\right)\right\}\right. \\
& \leqslant 3 C \lim _{k \rightarrow \infty} \max \left\{\frac{a_{k}}{\varphi_{0}\left(a_{k}\right)}, a_{k} \varphi_{1}\left(a_{k}\right)\right\}=0 .
\end{aligned}
$$

Соотношение(45) показывает, что для любого симметричного пространства $X$ найдется такая пара весов $\omega_{0}$ и $\omega_{1}$, что $K$-функционал и $K^{c}$-функционал равномерно неэквивалентны на некоторых конусах.

Покажем теперь, что на общем конусе множества

$$
\left(X\left(\omega_{0}\right), X\left(\omega_{1}\right)\right)_{K^{c}, \Phi_{\theta, q}}, \quad\left(X\left(\omega_{0}\right), X\left(\omega_{1}\right)\right)_{K, \Phi_{\theta, q}}
$$

с $0<\theta<1, q \geqslant 1$ имеют неэквивалентные нормы.

Из соотношений $(36)$ и $(40)$ следует, что если $x \in S(\mu)^{c}$ и $\operatorname{supp} x=\bigcup_{j=2 k}^{\infty} J_{j}$, то

$$
\frac{1}{3} \min (1, t)\left\|x\left|X\left\|\leqslant K^{c}\left(t, x ; X\left(\omega_{0}\right), X\left(\omega_{1}\right)\right) \leqslant \min (1, t)\right\| x\right| X\right\|
$$

Поэтому для $x \in S(\mu)^{c} \mathrm{c} \operatorname{supp} x=\bigcup_{j=2 k}^{\infty} J_{j}$ верны соотношения

$\frac{1}{3}\left\|\min (1, t)\left|\Phi_{\theta, q}\|\cdot\| x\right| X\right\| \leqslant\left\|x\left|\left(X\left(\omega_{0}\right), X\left(\omega_{1}\right)\right)_{K^{c}, \Phi_{\theta, q}}\|\leqslant\| \min (1, t)\right| \Phi_{\theta, q}\right\| \cdot\|x \mid X\|$.

Поскольку верно равенство

$$
\left\|\min (1, t) \mid \Phi_{\theta, q}\right\|=\left(\frac{1}{(1-\theta) \theta q}\right)^{1 / q}
$$

то для $x \in S(\mu)^{c}$ c supp $x=\bigcup_{j=2 k}^{\infty} J_{j}$ выполнены соотношения

$$
\begin{aligned}
\frac{1}{3}\left(\frac{1}{(1-\theta) \theta q}\right)^{1 / q}\|x \mid X\| & \leqslant\left\|x \mid\left(X\left(\omega_{0}\right), X\left(\omega_{1}\right)\right)_{K^{c}, \Phi_{\theta, q}}\right\| \\
& \leqslant\left(\frac{1}{(1-\theta) \theta q}\right)^{1 / q}\|x \mid X\|
\end{aligned}
$$


Положим

$$
\psi(t)=t \chi\left(0, a_{k}\right)+a_{k} \chi\left(a_{k}, 1\right)+t a_{k} \chi\left(1, \frac{1}{a_{k}}\right)+\chi\left(\frac{1}{a_{k}}, \infty\right) .
$$

Тогда из (43) получим справедливость для всех $x \in S(\mu)^{c} \mathrm{c} \operatorname{supp} x=\bigcup_{j=2 k}^{\infty} J_{j}$ неравенства

$$
K\left(t, x ; X\left(\omega_{0}\right), X\left(\omega_{1}\right)\right) \leqslant C\left\|\psi(t)\left|\Phi_{\theta, q}\|\| x\right| X\right\|
$$

где $C$ не зависит от $x$.

Простой подсчет показьвает, что

$$
\left\|\psi(t) \mid \Phi_{\theta, q}\right\| \leqslant\left(\frac{1}{(1-\theta) \theta q}\right)^{1 / q}\left(a_{k}^{(1-\theta) q}+a_{k}^{\theta q}\right)^{1 / q}
$$

Поэтому для всех $x \in S(\mu)^{c} \mathrm{c} \operatorname{supp} x=\bigcup_{j=2 k}^{\infty} J_{j}$ верно неравенство

$$
K\left(t, x ; X\left(\omega_{0}\right), X\left(\omega_{1}\right)\right) \leqslant C\left(\frac{1}{(1-\theta) \theta q}\right)^{1 / q}\left(a_{k}^{(1-\theta) q}+a_{k}^{\theta q}\right)^{1 / q}\|x \mid X\|
$$

Поскольку $a_{k} \rightarrow 0$ при $k \rightarrow \infty$, то, сравнивая (46) и (47), видим, что на общем конусе множества $\left(X\left(\omega_{0}\right), X\left(\omega_{1}\right)\right)_{K^{c}, \Phi_{\theta, q}}$ и $\left(X\left(\omega_{0}\right), X\left(\omega_{1}\right)\right)_{K, \Phi_{\theta, q}}$ с $q \geqslant 1,0<$ $\theta<1$ имеют неэквивалентные нормы.

Теперь мы рассмотрим пример интерполяционной пары пространств с весом, которая очень часто встречается в гармоническом анализе и для которой конструкции вещественного метода интерполяции, построенные по $K$-функционалу Питре и по $K^{c}$-функционалу, совпадают. Некоторые интерполяционные пространства этой пары описьвались в [15], [16].

Пусть снова $\mu$ есть мера Лебега. Как обьчно, через $L^{p, q}$ обозначим пространство Лоренца, состоящее из тех функций, для которых конечна величина

$$
\left\|x \mid L^{p, q}\right\|=\left(\int_{0}^{\infty}\left(t^{1 / p} x^{*}(t)\right)^{q} \frac{d t}{t}\right)^{1 / q}
$$

Хорошо известно [1], что $L^{p, p}=L^{p}$. В обшем случае $L^{p, q}-$ квазинормированное пространство, но при $p>1$ квазинорму можно заменить на норму, при введении которой $L^{p, q}$ становится банаховым пространством.

Для пространства $L^{p, q}$ положим $\left(L^{p, q}\right)^{c}=L^{p, q} \cap S(\mu)^{c}$, а для пространства $\left(L^{1}(u), L^{\infty}\right)_{K, \Phi \theta, q}$ соответственно

$$
\left(L^{1}(u), L^{\infty}\right)_{K, \Phi_{\theta, q}}^{c}=\left(L^{1}(u), L^{\infty}\right)_{K, \Phi_{\theta, q}} \cap S(\mu)^{c}
$$


TeOpema 7. Пусть $u(s)=s^{\alpha} u 1 \leqslant q \leqslant \infty, \quad 0<\theta<1,-1<\alpha<\frac{\theta}{1-\theta}$, $1 / p=(\alpha+1)(1-\theta)$.

Тогда с точностью до эквивалентных норм верно равенство

$$
\left(L^{1}(u), L^{\infty}\right)_{K^{c}, \Phi_{\theta, q}}=\left(L^{1}(u), L^{\infty}\right)_{K, \Phi_{\theta, q}}^{c}=\left(L^{p, q}\right)^{c},
$$

причем для $x \in S(\mu)^{c}$ при $1<q \leqslant \infty$ верны неравенства

$$
(\alpha+1)^{\theta+1 / q-1}\left\|x\left|L^{p, q}\|\leqslant\| x\right|\left(L^{1}(u), L^{\infty}\right)_{K^{c}, \Phi_{\theta, q}}\right\| \leqslant \frac{(\alpha+1)^{\theta+1 / q}}{(1+\alpha) \theta}\left\|x \mid L^{p, q}\right\|,
$$

а при $q=1$ выполнено равенство

$$
\frac{(\alpha+1)^{\theta}}{\theta}\left\|x\left|L^{p, 1}\|=\| x\right|\left(L^{1}(u), L^{\infty}\right)_{K^{c}, \Phi_{\theta, 1}}\right\| .
$$

ДоКАЗАТЕЛЬСТВо. В нашем случае $p>1$. Поэтому пространство $L^{p, q}$ является банаховым. Соотношение

$$
\left(L^{1}(u), L^{\infty}\right)_{K, \Phi_{\theta, q}}=L^{p, q}
$$

хорошо известно [16], откуда следует равенство

$$
\left(L^{1}(u), L^{\infty}\right)_{K, \Phi_{\theta, q}}^{c}=\left(L^{p, q}\right)^{c} .
$$

Докажем, что

$$
\left(L^{1}(u), L^{\infty}\right)_{K^{c}, \Phi_{\theta, q}}=\left(L^{p, q}\right)^{c} .
$$

Пусть $x \in S(\mu)^{c}$. Тогда из теоремы 4 получим

$$
K^{c}\left(t, x ; L^{1}(u), L^{\infty}\right)=\int_{0}^{\tau(t)} x(s) u(s) d s,
$$

где

$$
\tau(t)=((\alpha+1) t)^{1 /(\alpha+1)} .
$$

По определению

$$
\begin{aligned}
\left\|x \mid\left(L^{1}(u), L^{\infty}\right)_{K^{c}, \Phi_{\theta, q}}\right\| & =\left(\int_{0}^{\infty}\left(t^{-\theta} K^{c}\left(t, x, L^{1}(u), L^{\infty}\right)\right)^{q} \frac{d t}{t}\right)^{1 / q} \\
& =\left(\int_{0}^{\infty}\left(t^{-\theta} \int_{0}^{\tau(t)} x(s) u(s) d s\right)^{q} \frac{d t}{t}\right)^{1 / q} .
\end{aligned}
$$

Сделав замену переменных $s=\tau(t) \sigma$, получим

$$
\left\|x \mid\left(L^{1}(u), L^{\infty}\right)_{K^{c}, \Phi_{\theta, q}}\right\|=\left(\int_{0}^{\infty}\left(t^{-\theta} \int_{0}^{1} x(\sigma \tau(t)) \tau(t)(\sigma \tau(t))^{\alpha} d \sigma\right)^{q} \frac{d t}{t}\right)^{1 / q}
$$


Из равенства (50) следует, что

$$
\begin{aligned}
\| x \mid & \left(L^{1}(u), L^{\infty}\right)_{K^{c}, \Phi_{\theta, q}} \| \\
& =\left(\int_{0}^{\infty}\left(t^{-\theta} \int_{0}^{1} x(\sigma \tau(t)) t(1+\alpha) \sigma^{\alpha} d \sigma\right)^{q} \frac{d t}{t}\right)^{1 / q} \\
& =(\alpha+1)\left(\int_{0}^{\infty}\left(t^{1-\theta} \int_{0}^{1} x(\sigma \tau(t)) \sigma^{\alpha} d \sigma\right)^{q} \frac{d t}{t}\right)^{1 / q} \\
& =(\alpha+1)\left(\int_{0}^{\infty}\left(t^{1-\theta} \int_{0}^{1} x\left(\sigma[(\alpha+1) t]^{1 /(\alpha+1)}\right) \sigma^{\alpha} d \sigma\right)^{q} \frac{d t}{t}\right)^{1 / q}
\end{aligned}
$$

Далее, воспользуемся неравенством Минковского и получим

$$
\begin{aligned}
& \| x \mid\left(L^{1}(u), L^{\infty}\right)_{K^{c}, \Phi_{\theta, q} \|} \\
& \leqslant(\alpha+1) \int_{0}^{1}\left(\int_{0}^{\infty}\left(t^{1-\theta} x\left([(\alpha+1) t]^{1 /(\alpha+1)} \sigma\right) \sigma^{\alpha}\right)^{q} \frac{d t}{t}\right)^{1 / q} d \sigma=K_{1} .
\end{aligned}
$$

Отсюда с помощью замены переменных $\tau=[(\alpha+1) t]^{1 /(\alpha+1)} \sigma$ придем к соотношению

$$
\begin{aligned}
K_{1} & =(\alpha+1)^{\theta+1 / q} \int_{0}^{1}\left(\int_{0}^{\infty}\left[\tau^{(1-\theta)(\alpha+1)} x(\tau)\right]^{q} \frac{d \tau}{\tau}\right)^{1 / q} \sigma^{\theta(\alpha+1)-1} d \sigma \\
& =(\alpha+1)^{\theta+1 / q} \int_{0}^{1} \sigma^{\theta(\alpha+1)-1} d \sigma\left\|x \mid\left(L^{p, q}\right)^{c}\right\| \\
& =\frac{(\alpha+1)^{\theta+1 / q}}{\theta(\alpha+1)}\left\|x \mid\left(L^{p, q}\right)^{c}\right\| .
\end{aligned}
$$

Таким образом, мы получили

$$
\left\|x\left|\left(L^{1}(u), L^{\infty}\right)_{K^{c}, \Phi_{\theta, q}}\left\|\leqslant \frac{(\alpha+1)^{\theta+1 / q}}{\theta(\alpha+1)}\right\| x\right|\left(L^{p, q}\right)^{c}\right\| .
$$

Докажем обратное неравенство. Поскольку $x$ убывает, то согласно теореме 4 и (49), (50) получим

$$
\begin{aligned}
& \left\|x \mid\left(L^{1}(u), L^{\infty}\right)_{K^{c}, \Phi_{\theta, q}}\right\|=\left(\int_{0}^{\infty} t^{-\theta}\left(\int_{0}^{[(\alpha+1) t]^{1 /(\alpha+1)}} x(s) u(s) d s\right)^{q} \frac{d t}{t}\right)^{1 / q} \\
& \geqslant\left(\int_{0}^{\infty}\left(t^{-\theta} x\left([(\alpha+1) t]^{1 /(\alpha+1)}\right)\right)^{q}\left(\int_{0}^{[(\alpha+1) t]^{1 /(\alpha+1)}} u(s) d s\right)^{q} \frac{d t}{t}\right)^{1 / q} \\
& =\left(\int_{0}^{\infty}\left(t^{1-\theta} x\left([(\alpha+1) t]^{1 /(\alpha+1)}\right)\right)^{q} \frac{d t}{t}\right)^{1 / q} \text {. }
\end{aligned}
$$


Положив $s=[(\alpha+1) t]^{1 /(\alpha+1)}$, окончательно получим

$$
\left\|x \mid\left(L^{1}(u), L^{\infty}\right)_{K^{c}, \Phi_{\theta, q}}\right\| \geqslant(\alpha+1)^{1 / q+\theta-1}\left(\int_{0}^{\infty}\left(s^{(\alpha+1)(1-\theta)} x(s)\right)^{q} \frac{d s}{s}\right)^{1 / q} .
$$

Таким образом, справедливо и неравенство

$$
(\alpha+1)^{\theta+1 / q-1}\left\|x\left|\left(L^{p, q}\right)^{c}\|\leqslant\| x\right|\left(L^{1}(u), L^{\infty}\right)_{K^{c}, \Phi_{\theta, q}}\right\| .
$$

Объединяя (51) и (52), придем к (48). Для окончания доказательства в случае $q=1$ достаточно просто воспользоваться теоремой Фуббини. Теорема доказана.

\section{Список литературы}

1. Берг Й., Лефстрем Й. Интерполяционные пространства. Введение. М.: Мир, 1980.

2. Брудный Ю.А., Крейн С.Г., Семенов Е.М. Интерполяция линейных операторов // Итоги науки и техники. Сер. Матем. анализ. Т. 24. М.: ВИНИТИ, 1986. С. 3-164.

3. Brudniy Ju., Krugliak N. Interpolations Functors and Interpolations Spaces. North-Holland, 1991.

4. Крейн С. Г., Петунин Ю.И., Семенов Е.М. Интерполяция линейных операторов. М.: Наука, 1978.

5. Трибель X. Теория интерполяции, функциональные пространства, дифференциальные операторы. М.: Мир, 1980.

6. Мазья В. Г. Пространства С. Л. Соболева. Л.: Изд-во Ленингр. ун-та, 1985.

7. Самко С.Г., Килбас А. А., Маричев О. И. Интегралы и производные дробного порядка и некоторые их приложения. Минск: Наука и техника, 1987.

8. Бережной Е.И. Точные оценки операторов на конусах в идеальных пространствах // Тр. МИ РАН. 1993. Т. 204. С. 3-35.

9. Sawyer E.T. Boundedness of classical operators on classical Lorentz spaces // Studia Math. 1991. V. 96. P. 145-158.

10. Канторович Л. В., Акилов Г. П. Функциональный анализ. М.: Наука, 1977.

11. Lindenstrauss J., Tzafriri L. Classical Banach Spaces. 1, 2. N. Y.: Springer, 1973, 1979.

12. Бережной Е. И., Забрейко П. П. Интерполяция частично аддитивных операторов // ДАН БССР. 1986. Т. 30. № 2. С. 108-111.

13. Berezhnoy E. I., Zabreiko P. P. Some interpolation theory for nonlinear operators // Nonlinear Analysis. 1988. V. 12. № 2. P. 155-170.

14. Дмитриев В.И., Крейн С.Г., Овчинников В.И. Основы теории интерполяции линейных операторов // Геометрия линейных пространств и теория операторов. Ярославль, 1977. С. 31-75.

15. Лизоркин П. И. Интерполяция пространств $L^{p}$ с весом // ДАН СССР. 1975. Т. 222. № 1. C. 32-35.

16. Maligranda L., Persson L. Interpolation between weighted $L^{p}$ and Lorentz spaces // Bull. Pol. Acad. Sci. Math. 1987. V. 35. № 11-12. P. 765-768.

Ярославский университет

Математический институт им. В.А.Стеклова РАН

Поступило в редакцию

E-mail: smirn@delta.yaroslavl.su 